\title{
Theory of hierarchical coupling
}

\author{
A.I. Olemskoi ${ }^{\mathrm{a}, 1}$, A.B. Kiselev ${ }^{\text {b,2 }}$ \\ a Department of Physical Electronics, Sumy State University, 2, Rimskii-Korsakov St., 244007 Sumy, Ukraine \\ b Department of Pure and Applied Mathematics, Chernigov Technological Institute, 95, Shevchenko St., 250027 Chernigov, Ukraine
}

Received 5 March 1998; revised manuscript received 25 June 1998; accepted for publication 14 July 1998

Communicated by A.P. Fordy

\begin{abstract}
A recursion relation between the intensity of hierarchical objects at neighbouring levels of a hierarchical tree, the strength of the coupling between them and the level distribution of nodes of the hierarchical tree are proposed. Regular (including Fibonacci), degenerate and irregular trees are considered. It is shown that the strength of hierarchical coupling is an exponentially, logarithmically or power law decreasing function of distance from a common ancestor, respectively. (C) 1998 Elsevier Science B.V.
\end{abstract}

PACS: 47.53.+n; 64.60.Ak; 89.90.+n

Keywords: Hierarchical trees; Ultrametric space; Recursion relation

\section{Formulation of the problem}

Despite the widespread occurrence of hierarchy in social life and its importance to other systems [1], the theory of hierarchically subordinated ensembles has mainly evolved in order to understand the dynamics of spin glasses $[2,3]$. The key point is that the hierarchically subordinated objects form an ultrametric space. Geometrically the latter can be conceived of as a Cayley tree (see Fig. 1). The degree of hierarchical coupling between objects $w$, corresponding to the nodes of a given level, depends on the distance between them defined by the number of steps $m$ to a common ancestor. So the ultrametric space is equipped with metrics, $\zeta \propto m$ ( $\zeta$ is the distance). The primary goal of this work is to show how the function $w(\zeta)$ can be derived for different types of hierarchical trees.

\footnotetext{
${ }^{1}$ E-mail: alexander@olem.sumy.ua.

${ }^{2}$ E-mail: adk@kid.ti.chernigov.ua.
}

Let $z_{k}$ be the intensity of a hierarchical object at level $k$. We assume that the intensity $z_{k}$ increases by going from level $k$ to the nearest higher level $k-1$ (it looks like climbing the career ladder). Mathematically, it can be expressed in terms of the simple recursion relation

$z_{k-1}=z_{k}+N_{k}^{-1} w\left(z_{k}\right)$,

where $N_{k}$ is the number of nodes at level $k$ and $w\left(z_{k}\right)$ is the required function of hierarchical coupling. In the case of regular tree, shown in Fig. 1a, we have the exponentional dependence of $N_{k}$ on $k$ which is typical of fractal objects,

$N_{k}=j^{k}$,

where $j$ is the branching ratio of the tree (the case of $j=2$ is shown in Fig. 1a). Another example is the degenerate tree with only one branching node per level (see Fig. 1b), so that 

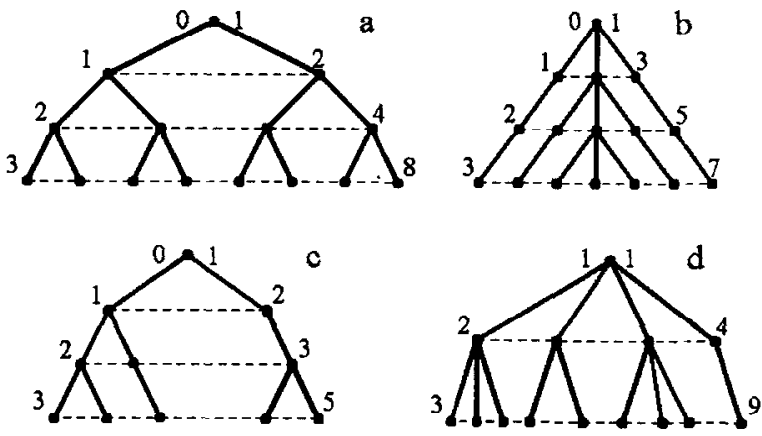

Fig. 1. Basic types of hierarchical trees (the level number is indicated at the left, the corresponding number of nodes at the right): (a) regular tree with $j=2$; (b) degenerate tree with $j=3$; (c) Fibonacci tree; (d) irregular tree for $n=1$ at $a=2$.

$N_{k}=(j-1) k+1$.

For the Fibonacci tree (Fig. Ic), the number of nodes on the $k$ th level, $N_{k}=F(k+2)$, is determined by the Fibonacci numbers $F(k), k=0,1, \ldots$, which obey the equation $F(k+2)=F(k+1)+F(k)$ with $F(1)=$ $F(2)=1$. For $k \gg 1$ the latter implies that $F(k+2) \approx$ $q \tau^{k}$, where $q \approx 1.17082$ and $\tau=(\sqrt{5}+1) / 2 \approx$ 1.61803 is the golden mean. In view of this, the number of nodes of the $k$ th level of the Fibonacci tree is

$N_{k} \approx q \tau^{k}, \quad k \gg 1$

Lastly, for the irregular tree like that depicted in Fig. 1d a power law approximation of the form

$N_{k} \approx n k^{a}, \quad n>0, \quad a<1$

is suggested.

Note that Eq. (1) was originally used for describing a resonance hierarchy in nonlinear oscillations [4] and enjoys the property of self-similarity that is a distinguishable feature of hierarchical systems. In order to clarify the point, suppose that $z_{k} \propto \xi^{k}$ with $\xi \leqslant 1$ being the similarity parameter and $w(z)$ is a homogeneous function, $w(\xi z)=\xi^{\alpha} w(z)$. Then in the limiting case $k \gg 1$, when $z_{k-1} \sim z_{k}$, Eqs. (1), (2) give the conventional relation

$\alpha=1-D, \quad D \equiv \ln j / \ln \xi^{-1}$,

linking the exponent $\alpha$ of a physical quantity with the fractal dimension $D \leqslant 1$ of a self-similar type object in the sense of the rugged coastline [5]. Moreover, since the function $w(z)$ can be assumed to be of the form $w(z)=W z^{\alpha}$ where $W$ is a positive constant, substituting the expression $z_{k}=x_{k} \xi^{k}=x_{k} j^{-k / D}$ in Eqs. (1), (2) provides the recursion relation for $x_{k}$ of the following form,

$x_{k-1}=\phi\left(x_{k}\right), \quad \phi(x) \equiv \xi\left(x+W x^{1-D}\right)$.

The map $\phi(x)$ has two fixed points: the stable one $x_{s}=0$ and the critical one

$x_{c} \equiv\left(\frac{W}{j^{1 / D}-1}\right)^{1 / D}$.

As a result of this, we obtain the following homogeneous functions,

$z_{k}=x_{c} j^{-k / D}$.

$w_{k}=W^{1 / D}\left(j^{1 / D}-1\right)^{-\Delta} j^{-\Delta k}$,

where

$\Delta=(1-D) / D$

is the decrement that determines the scale of hierarchical coupling in ultrametric space.

\section{Recursion relation in the continuum approximation}

When $k \rightarrow \infty$ the continuum approximation can be used, so that the finite difference $z_{k}-z_{k-1}$ is replaced with the derivative $\mathrm{d} z / \mathrm{d} k$. Eq. (1) then can be rewritten in the integral form,

$\int \frac{\mathrm{d} z}{w(z)}=-\int \frac{\mathrm{d} k}{N_{k}} \equiv(\ln j)^{-1} \int \frac{\mathrm{d} \zeta}{N(\zeta)}$,

$\zeta \equiv\left(k_{0}-k\right) \ln j, \quad k \leqslant k_{0}$,

where $\zeta$ is the distance in ultrametric space and $k_{0} \gg$ 1 is the number of the hierarchical level under consideration. Since the main contribution to the integral on the left-hand side of Eq. (12) is determined by small $z$ we just need to know the asymptotical behavior of $w(z)$ at $z \rightarrow 0$. According to the above discussion, for small $z$ the function $w(z)$ is homogeneous and can be taken to be of the form

$w(z)=W z^{1-D}, \quad z \rightarrow 0$. 
It is not difficult then to solve Eq. (12) for different trees with the node numbers $N_{k}$ defined by Eqs. (2)(5) and with Eq. (13) taken into consideration.

\subsection{Regular and Fibonacci trees}

In the case of a regular tree the dependences of level intensity $z(\zeta)$ and strength of hierarchical coupling $w(\zeta)$ on the distance $\zeta$ in ultrametric space are as follows,

$z=W^{-1 /(1-D)}\left[(1-u)+u \mathrm{e}^{\zeta-\zeta_{0}}\right]^{1 / D}$,

$u \equiv D W^{1 /(1-D)} / \ln j, \quad \zeta_{0} \equiv k_{0} \ln j$,

$w=\left[(1-u)+u \mathrm{e}^{\zeta-\zeta_{0}}\right]^{4}, \quad \zeta \leqslant \zeta_{0}$.

In Eq. (16) and hereafter $w(\zeta)$ is assumed to satisfy the condition $w\left(\zeta_{0}\right)=1$. So, if the distance $\zeta$ to a common ancestor increases, the functions $z(\zeta)$ and $w(\zeta)$ reveal exponential increase with increments $D^{-1}$ and $\Delta$ correspondingly. The increment of hierarchical subordination amplification (11) becomes zero for the system with $D=1$ (from Eq. (14) the latter means ideal hierarchical subordination). Both of the decrements $D^{-1}$ and $\Delta$ increase indefinitely as the fractal dimension $D$ decreases to zero.

Starting from Eq. (7), it is straightforward to analyse solutions of Eq. (1) and to make a comparison between the results obtained in the continuum limit and exact ones. Referring to Fig. 2, where the graphs of functions $\phi(x)$ and $\phi^{-1}(x)$ are shown, it is seen that if the initial value $x_{0}$ obeys the condition $x_{0}<x_{c}$, just a few steps are needed for $x$ to approach zero. When $x_{0}=x_{c}$, the solution is defined by Eq. (8) and in the case of $x_{0}>x_{c}$ solutions increase indefinitely. It can be shown that in the latter case $z_{k}$ decays exponentially to a constant. At this stage, there is a good agreement between the qualitative conclusions of the continuum approximation and exact ones (see Fig. 3). It is interesting to note that Eq. (15) can be derived from the exact solution given by Eq. (9) only if $j^{1 / D}-1 \approx$ $\ln j / D$, which corresponds to the limiting case where $\ln j \ll D$. In order to clarify the above points note that within the framework of the continuum approximation the recursion relation (7) takes the form of well-known Landau-Khalatnikov equation

$\frac{\mathrm{d} x_{k}}{\mathrm{~d} k}=-\frac{\partial V}{\partial x_{k}}$,

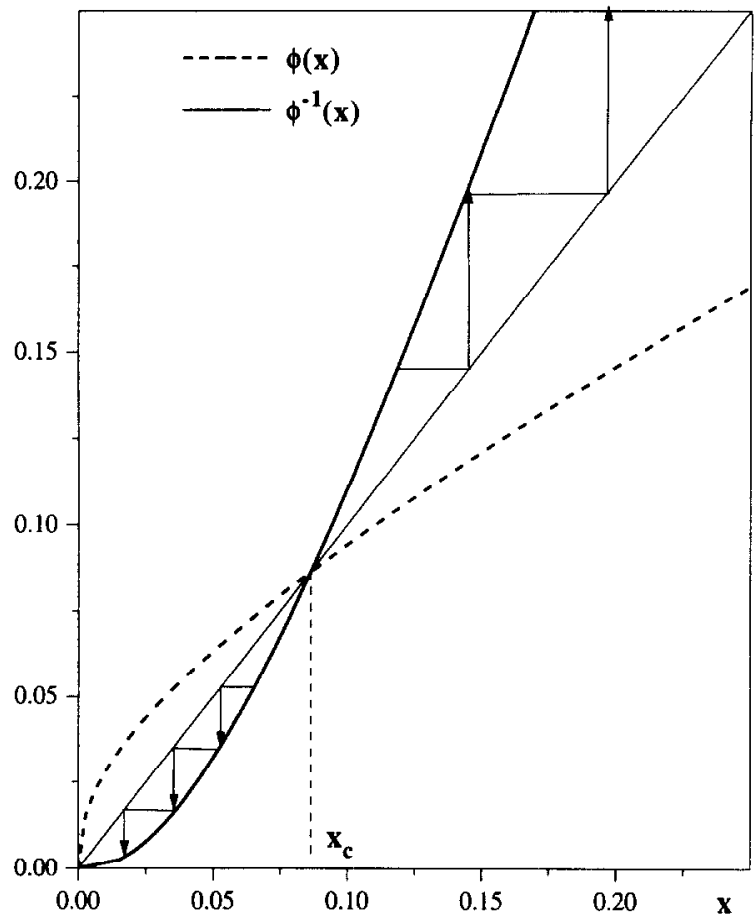

Fig. 2. The plots of $\phi(x)$ (dashed line) and $\phi^{-1}(z)$ (solid line) at $W=0.5$ and $D=0.6$ (the behaviour of $x$ under successive iterations of the map $\phi^{-1}(x)$ is shown by arrows for $x_{0}<x_{c}$ and $x_{0}>x_{c}$ ).

$V \equiv-\frac{x^{2}}{2}+\int \phi(x) \mathrm{d} x$,

where $V$ is the effective potential. Inserting the dependence $\phi(x)$ from Eqs. (7) into Eq. (18) yields the expression for the function $V(x)$,

$V=\frac{W j^{-1 / D}}{2-D} x^{2-D}-\frac{1-j^{-1 / D}}{2} x^{2}$,

where the second equation (6) is taken into account. According to Fig. 4, the potential $V(x)$ increases in the region $x<x_{c}$, where the value $x_{c}$ is given by Eq. (8), and then decreases indefinitely. It is noteworthy to point out the analogy with the theory of creation and growth of new phase precipitations, where at a precritical size of embryos the surface tension results in an increase of free energy which then decreases indefinitely due to thermodynamical stimulus of phase transition [6].

So, one can speak about instability of the hierarchical system with respect to the increase in the level number when the initial intensity $x_{0}$ exceeds the criti- 


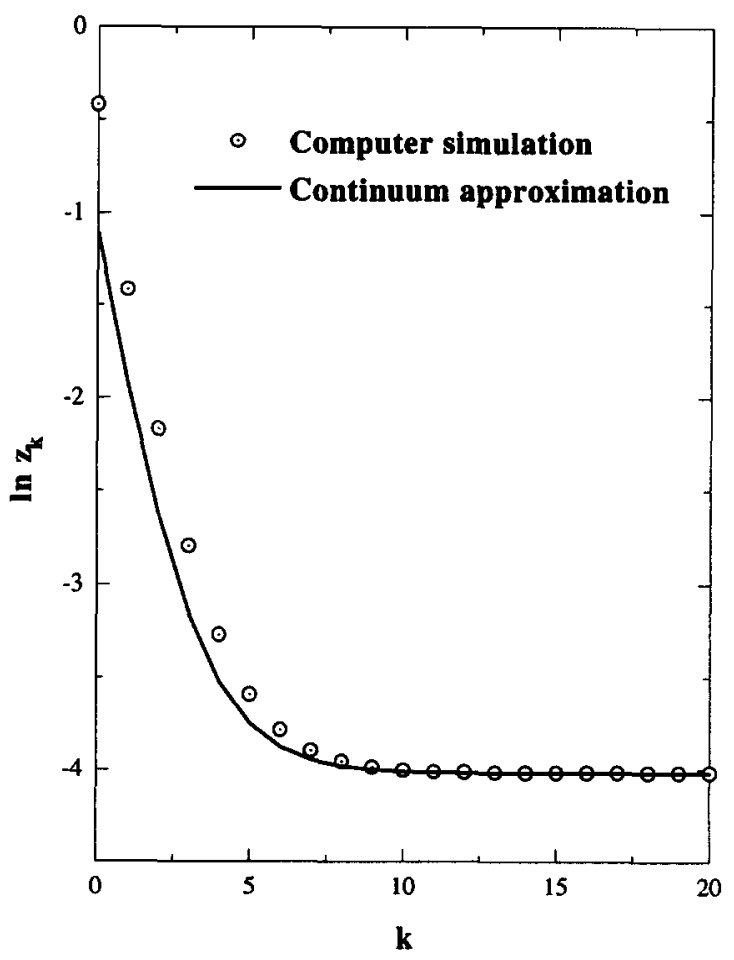

Fig. 3. The $k$-dependences of $\ln z_{k}$, where the solutions of Eq. (7) are obtained numerically (circle dots) and in the continuum approximation (solid line) at $W=1, j=2$ and $D=\ln j=0.6931$.

cal value $x_{c}$. This represents the well-known fact of the self-reproduction of bureaucracy in social hierarchy.

Note that the solution of Eq. (17) gives $z_{k}$ in the form

$$
\begin{aligned}
& z_{k}=\left(z_{0}^{D}-x_{c}^{D}\left(1-\mathrm{e}^{-D(1-\xi) k}\right)\right)^{1 / D} \mathrm{e}^{(1-\xi+\ln \xi) k}, \\
& \xi \equiv j^{-1 / D},
\end{aligned}
$$

that is equivalent to Eq. (15) when the abovementioned condition $\ln j \ll D$ is met, so that $\ln j \approx$ $j-1, \ln \xi \approx \xi-1$.

A comparison between Eq. (2) and Eq. (4) shows that for sufficiently large $k$ the case of the Fibonacci tree (Fig. 1c) can be reduced to the above-considered regular tree with $j$ and $W$ replaced by $\tau$ and $W / q$, respectively. So the fractal dimension $D$ is fixed and equals $\ln \tau / \ln 2 \approx 0.6942$ [7].

\subsection{Degenerate tree}

In the case of a degenerate tree, where $N_{k}$ is defined by Eq. (3), Eqs. (12), (14) give

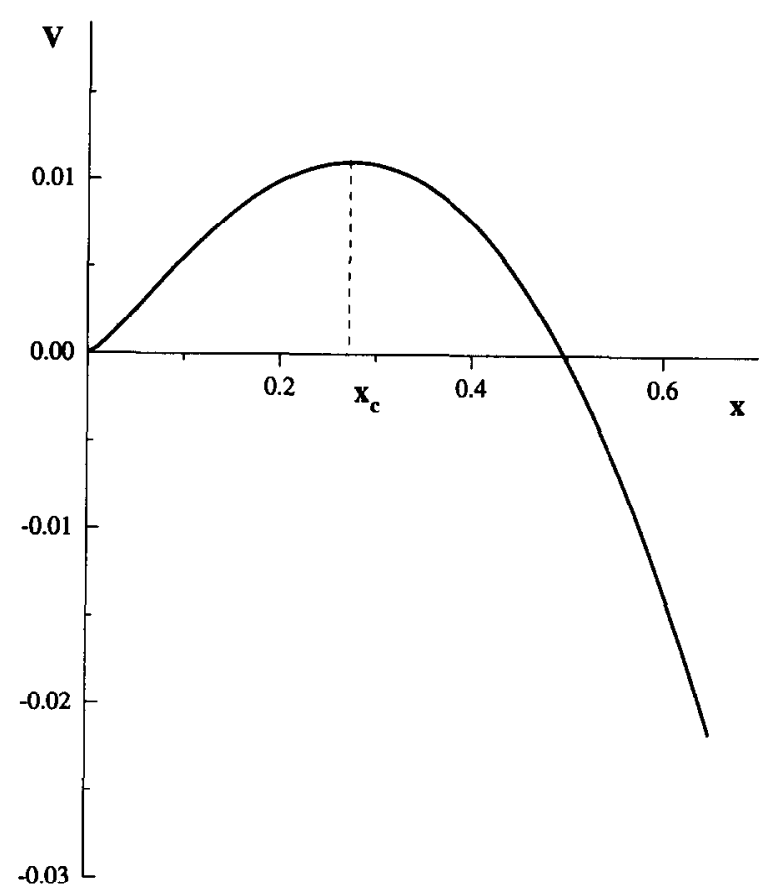

Fig. 4. The effective potential (19) as a function of $x$ at $W=1$, $j=2$ and $D=0.6$.

$z=W^{-1 /(1-D)}\left[1-u \ln \left(1+\frac{j-1}{\ln j}\left(\zeta_{0}-\zeta\right)\right)\right]^{1 / D}$,

$u \equiv \frac{D W^{1 /(1-D)}}{j-1}$,

$w=\left[1-\ln \left(1+\frac{j-1}{\ln j}\left(\zeta_{0}-\zeta\right)\right)\right]^{\Delta}$

When this result is compared with that of Eqs. (15), (16), it is apparent that going from the regular tree to degenerate one results in logarithmic slowing down of the foregoing exponential amplification of hierarchical subordination.

\subsection{Irregular tree}

Let us consider the intermediate case of a tree characterized by a power law growth in level number (Eq. (5), Fig. 1d). The result can be written as follows,

$$
\begin{aligned}
& z=W^{-1 /(1-D)}\left[1-u\left(1-\zeta / \zeta_{0}\right)^{1-a}\right]^{1 / D}, a<1, \\
& u \equiv \frac{D W^{1 /(1-D)} k_{0}^{1-a}}{n(1-a)}
\end{aligned}
$$


$w=\left[1-u\left(1-\zeta / \zeta_{0}\right)^{1-a}\right]^{\Delta}$.

From Eqs. (23), (24) it is clear that the level intensity and the strength of hierarchical subordination show a power law dependence on the distance $\zeta$.

\section{Discussion}

The above consideration shows both the level intensity $z$ and the strength of hierarchical coupling $w$ decrease as the level number $k$ increases and, conversely, they are increasing functions of the distance $\zeta$ in ultrametric space. In this connection, it should be emphasized that the dependence $w_{k}$ characterizes the degree of alliance of hierarchical objects at the reference level, whereas $w(\zeta)$ corresponds to the strength of hierarchical subordination.

A distinguishing feature of the regular tree is the fastest rate of change of $z(\zeta)$ and $w(\zeta)$. As it is seen from Eq. (10), the number of hierarchically subordinated level is bounded by the finite value

$\kappa=(\Delta \ln j)^{-1} \equiv D[(1-D) \ln j]^{-1}$.

In other words, the depth of hierarchical subordination $\kappa$ is finite for a regular tree, and in this sense it implies the weak hierarchical coupling. However, for the special case of totalitarian hierarchy, where the hierarchical coupling is ideal (in Eq. (14) $D=1$ ), the depth of the subordination is infinitely large $(\kappa=\infty)$. Nevertheless, according to Eq. (9) the intensity of the hierarchical objects still decays exponentially and the totalitarian system, though being ideally subordinated, is doomed to inefficiency. Social experiments that lend support to this conclusion are well known.

With passage to an irregular tree, which is supposed to be of widespread occurrence in nature, instead of exponentially fast decay inherent to a regular tree the hierarchical coupling exhibits a power law dependence. The slowest, logarithmic law corresponds to a degenerate hierarchy with only one branching node per level and can be realized as a selection system. For both irregular and degenerate trees the depth of hierarchical subordination is infinite, and the hierarchical coupling of such trees is strong.

It should be emphasized that the above strength of hierarchical coupling is fixed with the condition $w\left(\zeta_{0}\right)=1$ related to the top level. Since any real hier- archical system is usually built up from the top downwards, such a choice of normalization is preferred to the condition $w(\zeta=0)=1$. It seems, that given the choice the expressions inside the square brackets in Eqs. (15), (16), (23), (24) may take negative values at $\zeta=0$ under the parameter $u>1$. But since we are interested in the supercritical case where $z_{0}>x_{c}$ and, as is seen from comparison between Eq. (15) and Eq. (21), $u \equiv\left(x_{c} / z_{0}\right)^{D}$, the condition $u<1$ is fulfilled.

As is evident from the foregoing, the fractal dimension $D$, that governs the force of hierarchical coupling $w(z)$ at given configuration of a tree, plays an important part in the theory. Throughout this paper, it was adopted that the force of the hierarchical coupling is characterized by the only single value of $D$. It is not difficult to extend the consideration to the case of multifractal coupling. To do this one has to introduce an additional parameter $q \in(-\infty, \infty)$, so that the strength $w_{q}(z)$ is distributed over $q$ with density $\rho(q)$ and the fractal dimension $D(q)$ is in the range between the maximum dimension $D_{-\infty}$ and the minimum one $D_{+\infty}$ [7]. For example, $\rho$ for ideal hierarchical coupling is given by

$\rho(q)=\left|D^{\prime}\left(q_{0}\right)\right|^{-1} \delta\left(q-q_{0}\right)$,

where the prime denotes the derivative with respect to $q$ and $q_{0}$ is a solution of equation $D(q)=1$. As a result, the total strength is defined by

$w(\zeta)=\int_{-\infty}^{\infty} w_{q}(\zeta) \rho(q) \mathrm{d} q$,

where Eqs. (16), (22), (24) with $D$ replaced by $D(q)$ can be used as a kernel of Eq. (27). The dependences $D(q)$ and $\rho(q)$ for a given multifractal can be found after solving the respective problem (see Ref. [7]).

\section{Acknowledgement}

A.I.O. is grateful to the International Science Foundation for financial support under the Grant SPU072044 (ISSEP). 


\section{References}

[1] J.S. Nicolis, Dynamics of Hierarchical Systems: An Evolutionary Approach (Springer, New York, 1986).

[2] M. Mézard, G. Parisi, M.A. Virasoro, Spin Glass Theory and Beyond (World Scientific, Singapore, 1987).

[3] R. Rammal, G. Toulouse, M.A. Virasoro, Rev. Mod. Phys. 58 (1986) 765 .
[4] N.M. Krylov, N.N. Bogoliubov, Introduction to Nonlinear Mechanics (Acad. Sci. USSR, Kiev, 1937) [in Russian].

[5] E. Feder, Fractals (Plenum Press, New York, 1989).

[6] L.D. Landau, E.M. Lifshits, Statistical Physics, Part 1 (Pergamon Press, Oxford, 1980).

[7] A.I. Olemskoi, Fractals in Condensed Matter Physics, in: Physics Reviews, Vol. 18, Part 1, I.M. Khalatnikov, ed. (Gordon and Breach, London, 1996). 